BNL - 47416

Informal Report

APPLICATION OF MULTITRACER TECHNOLOGY TO PETROLEUM RESERVOIR STUDIES

QUARTERLY PROGRESS REPORT

REPORTING PERIOD: JANUARY 1 - MARCH 31, 1992

G. I. SENUM

APRIL 1992

DEPARTMENT OF APPLIED SCIENCE

BROOKHAVEN NATIONAL LABORATORY UPTON, LONG ISLAND, NEW YORK 11973

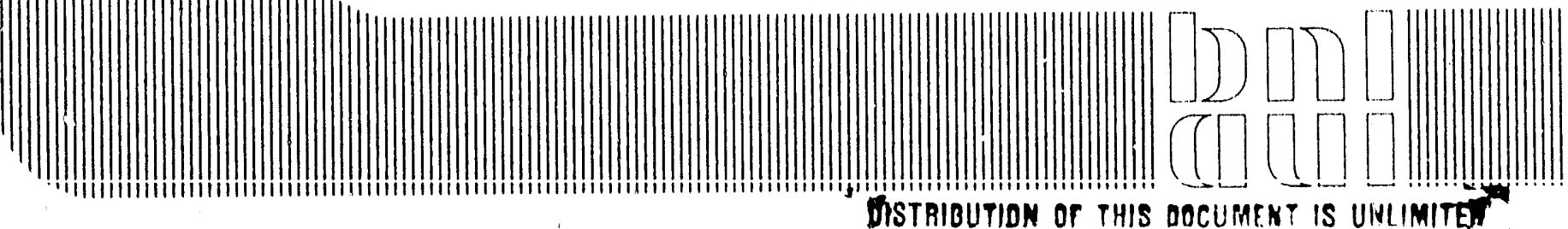


BNL---47416

DE92 014688

\title{
APPLICATION OF MULTITRACER TECHNOLOGY TO PETROLEUM RESERVOIR STUDIES
}

\author{
QUARTERLY PROGRESS REPORT
}

REPORTING PERIOD: JANUARY 1 - MARCH 31, 1992.

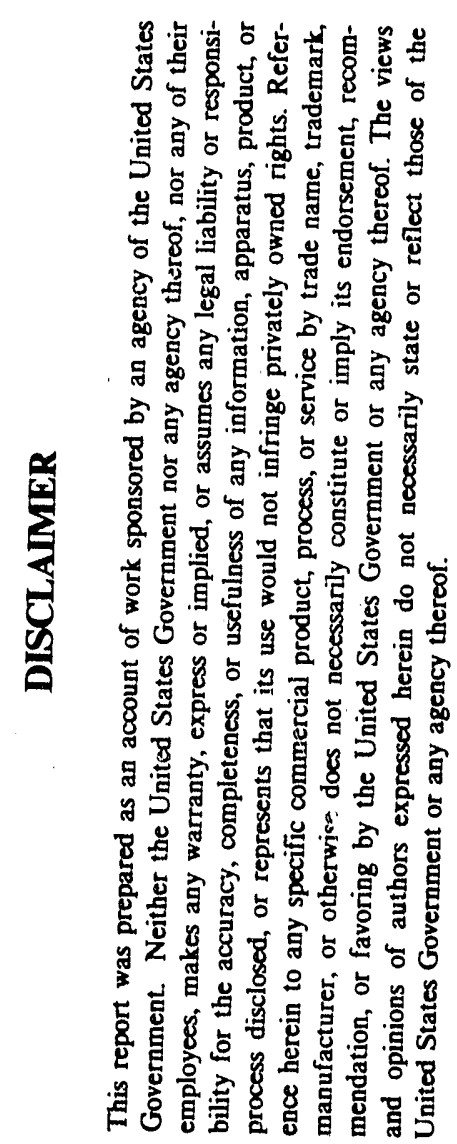

\section{MASTER}

Prepared for

Chandra M. Nautiyal, Project Manager

Bartlesville Project Office

U.S. Department of Energy

Bartlesville, OK 74005 


\section{DISCLAIMER}

This report was prepared as an account of work sponsored by the United States G vernment. Neither the United States nor the United States Department of Energy, nor any of their employees, nor any of their contractors, subcontractors, or their employees, makes any warranty, express or implied, or assumes any legal liability or responsibility for the accuracy, completeness, or usefulness of any information, apparatus, product or process disclosed, or represents that its use would not infringe privately owned rights. 


\section{OBJECTIVES}

The objectives of this research program are to:

1. Improve the assessment of the character of petroleum reservoirs using tracer technology for the monitoring and improvement of EOR techniques, specifically, (a) to apply the presently available multitracer perfluorocarbon tracer (PFT) technology to the study of petroleum reservoirs in characterizing reservoir bulk subsurface flow transport and dispersion rates; and, (b) to demonstrate that PFTs with differing physical properties will interact with differing rates of adsorption and dispersion within such reservoirs, from which may be inferred difference in the character and/or extent of petroleum in those reservoirs.

2. To develop a data base of petroleum transport and dispersion properties from tracer experiments for use by modellers for developing, validating and extending petroleum reservoirs models used for characterizing petroleum reservoirs.

\section{SUMMARY OF TECHNICAL PROGRESS}

Technical progress will be discussed according to the three ongoing field experiments at the Naval Petroleum Reserve in California (NPRC).

\section{The Shallow Oil Zone (SOZ) Experiment}

The SOZ is a multilayered reservoir which originally contained more than 1,250 million barrels of oil. It is estimated that the primary recovery efficiency in the SOZ will be less than $50 \%$. Cumulative production to date is $439 \mathrm{MMBO}$. The SS-1 interval in the SOZ has the lowest reservoir pressure, less than $50 \mathrm{psig}$, of any of the intervals in the SOZ. The low reservoir pressure provides the opportunity to increase the economic value of its remaining primary reserves by implementing processes which increase reservoir pressure and productivity, thereby accelerating recovery. 
Gas injection is a potentially viable means to increase the economic value of the remaining oil-in-place by supplementing the natural gravity drainage mechanism. A pilot gas injection project was initiated in April 1991 to gather production and injection data to assess the technical feissibility and economic viability of injecting gas to increase reservoir pressure and productivity.

The PFTs were specifically utilized to qualitatively determine the direction of migration of the injected gas and possible gas migration across the faults. PMCP and PMCH were simultaneously injected into $34-10 \mathrm{G}$ on June 18,1991 over a 28 hour period when the well was injecting 25 () MCFPD of gas. The PFTs were introduced into the reservoir in the gas phase by injecting a small flow of PFTs from a high pressure cylinder flowing through a regulator into the injection well. The regulator was used to ensure a constant injection rate over the 28 hour injection period. The high pressure cylinder contained approximately $0.05 \%$ of gaseous PMCP and $0.06 \%$ of gaseous $\mathrm{PMCH}$ in nitrogen. The resulting PFT concentration in the $34-10 \mathrm{G}$ injection gas was 89,330 picoliters of PMCP per liter of injection gas and 97,000 picoliters of $\mathrm{PMCH}$ per liter of injection gas. Likewise, oc-PDCH $(64,700 \mathrm{pL} / \mathrm{L})$ and ot-PDCH $(139,000 \mathrm{pL} / \mathrm{L})$ were injected into $44-10 \mathrm{G}$ on June 19, 1991 over a 24 hour period when the well was injecting 75 MCFPD. Approximately 12 grams of each PFT was used in this experiment. Ten monitoring wells (including both inactive gas cap wells and active producers) were sampled daily starting on June 19, 1991. The gas cap wells were produced at low rates, about $1 \mathrm{MCFPD}$, to ensure turnover of the gas in the well bore. Samples were collected directly at the well head into pre-evacuated one liter pressure bombs by the field operator.

Fifty $\mathrm{mL}$ aliquots of gas were withdrawn from the pressure bombs and injected onto CATS (Capillary Adsorbent Tracer Samplers) and sent to BNL for analysis for PFTs by gas chromatography and electron capture detection.

In this quarter, PFT injected at 44-10G finally broke through at $25-10 \mathrm{G}$, as is shown in Figure 1. Based on this observation the sampling program was reevaluated and several wells were deleted and others added in order to determine where the PFT in 44-10G was being transportecl. Sampling is continuing at these wells. 
Also, PFT injected at $34-10 \mathrm{G}$ continued to breakthrough at $81-10 \mathrm{G}$, a well located 3600 feet from the injector. The PFT concentrations were comparable to those detected at 24-10G (five days after injection) indicating a more or less equal flow of injected gas in the opposite direction. The two PFTs shown a retardation effect due to the PFT partitioning in the residual pore oil along the path of transport. This measured retardation will allow an estimation of the residual pore oil saturation to be made.

Several wells outside the sampling area have been occasionally spot-checked for tracer in this quarter with unexpected tracer detection in one well. This has lead to additional spot-checking of wells for PFT in order to fully discern the injected gas flow in SOZ.

This experiment is entering a period of a low level of activity, since the primary goals for this experiment appear to have been met. Some conclusions can tentatively be drawn from the results of the PFT study:

1) Detection of PFT in wells outside of the pilot project area confirmed that the faults bounding the pilot project are not absolutely sealing, but are restrictors to the migration of injected gas at low reservoir pressures (10 to $20 \mathrm{psig}$ ). Analysis of incremental reservoir pressures in the pilot indicates the bounding faults are leaking approximately $10 \%$ of the injected gas.

2) Explanation of the observed PFT migration patterns in the reservoir may require re-interpretation of the current fault patterns. The detection of PFT in well 81-10G, but not in well 78-3G, and the confirming reservoir pressure data, indicates that a potentially different fault orientation exists.

3) Areas of production response are consistent with increasing detection of PFTs.

4) The observed retardation between the arrival of the two PFTs at 81-10G and 25-10G may possibly provide an estimate of residual pore oil saturation along the path from the injection wells to these monitoring wells. 


\section{The 24Z Experiment}

The $24 \mathrm{Z}$ reservoir at NPRC is currently undergoing gas and water injection for pressure maintenance. The gas injection is into the gas cap and the water injectors are at the edges of the $24 \mathrm{Z}$ field. A six PFT experiment was started in mid-October/mid-November in this field. Two PFTs (PMCP and PMCH) were injected in the gas cap over a two week period. The PFT (about $30 \mathrm{gm}$ ) were dissolved in 500 gallons of methanol and injected at a proportional rate over two weeks. Four other PFTS (PDCB, o-PDCH, m-PDCH, and PTCH) were similarly injected into 4 water injectors, also over a two week duration.

Biweekly sampling started at the production wells in mid-November and, to date, there has been only one possible breakthrough of tracer, namely, the PFTs injected in the gas cap to a nearby producer. It is expected that this experiment will be of a longer duration than the SOZ, since it is estimated that the water injectors will breakthrough at the producers in a year.

\section{The 26R Experiment}

The 26R reservoir is currently undergoing gas injection for pressure maintenance. Six stages of tracer experiments are scheduled for this reservoir in order to discern the flow of injected gas in order to optimize the injected gas usage.

Stage 1 of the experiment was started in mid-November 1991, with two PFTs (PMCP and o-PDCH) injected at a gas injector, along with $\mathrm{SF}_{6}$, a field-proven tracer. The inclusion of $\mathrm{SF}_{6}$ is to provide a comparison of the PFTs with $\mathrm{SF}_{6}$. The specific goal of this stage 1 experiment is to uncover if the injected gas is crossing a certain fault. To date, no PFT has been detected at the twenty sampling wells.

In support of this 26R experiment, Gary Pope at UT/Austin has provided some modeling of this reservoir which indicated that tracer breakthrough should start to occur in April. Sampling will continue.

Stage III of this experiment was started in mid-March 1992, with the injection of PMCH. Sampling will start in April at surrounding production wells, some of which are in the Stage I sampling protocol. Breakthrough is not expected for several months for this stage. 


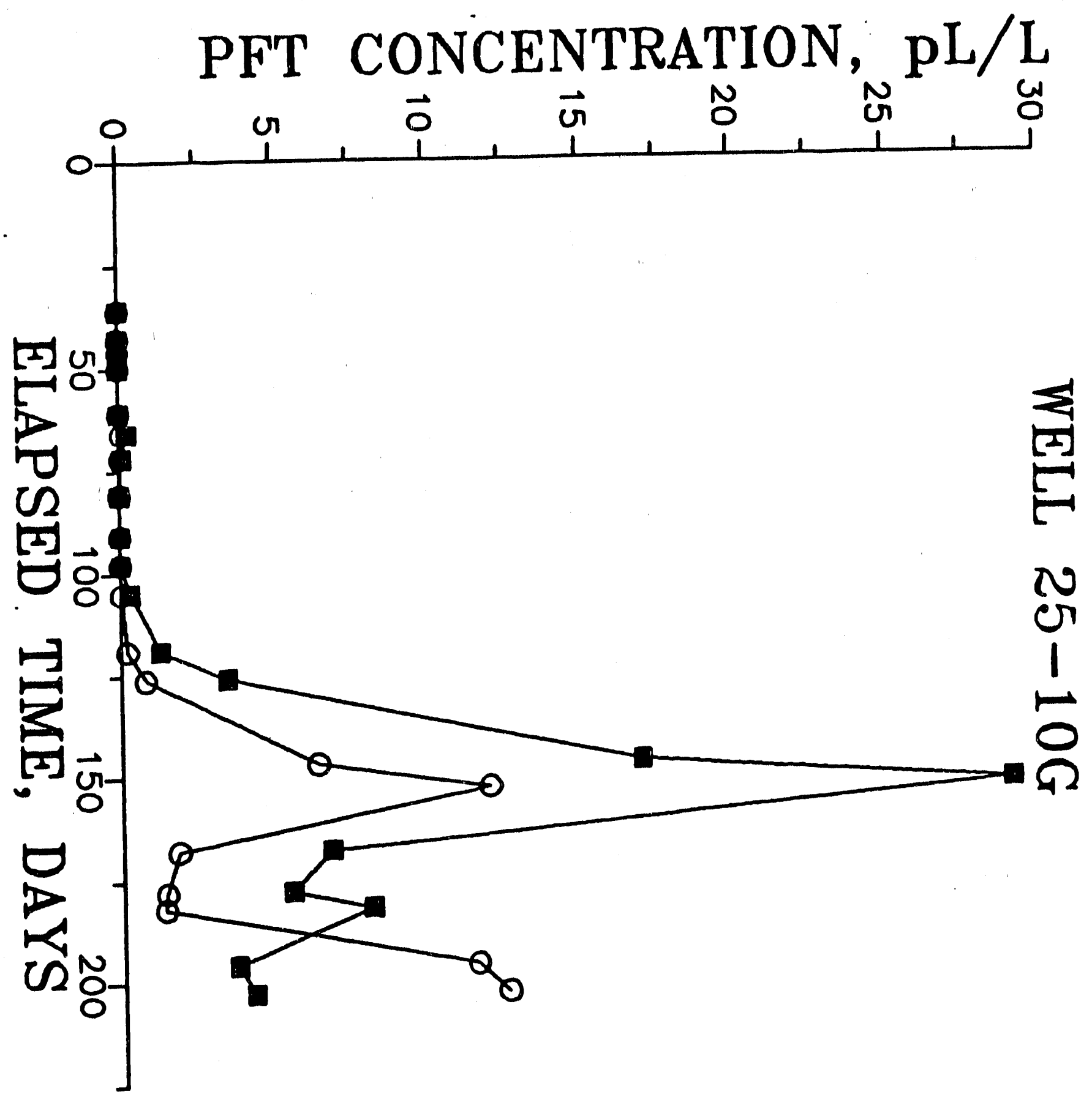

FIGURE MEASURED PFT CONCENTRATION AT WELL 25-10G; BOXES ARE oc-PDCH, CIRCLES ARE ot-PDCH. 

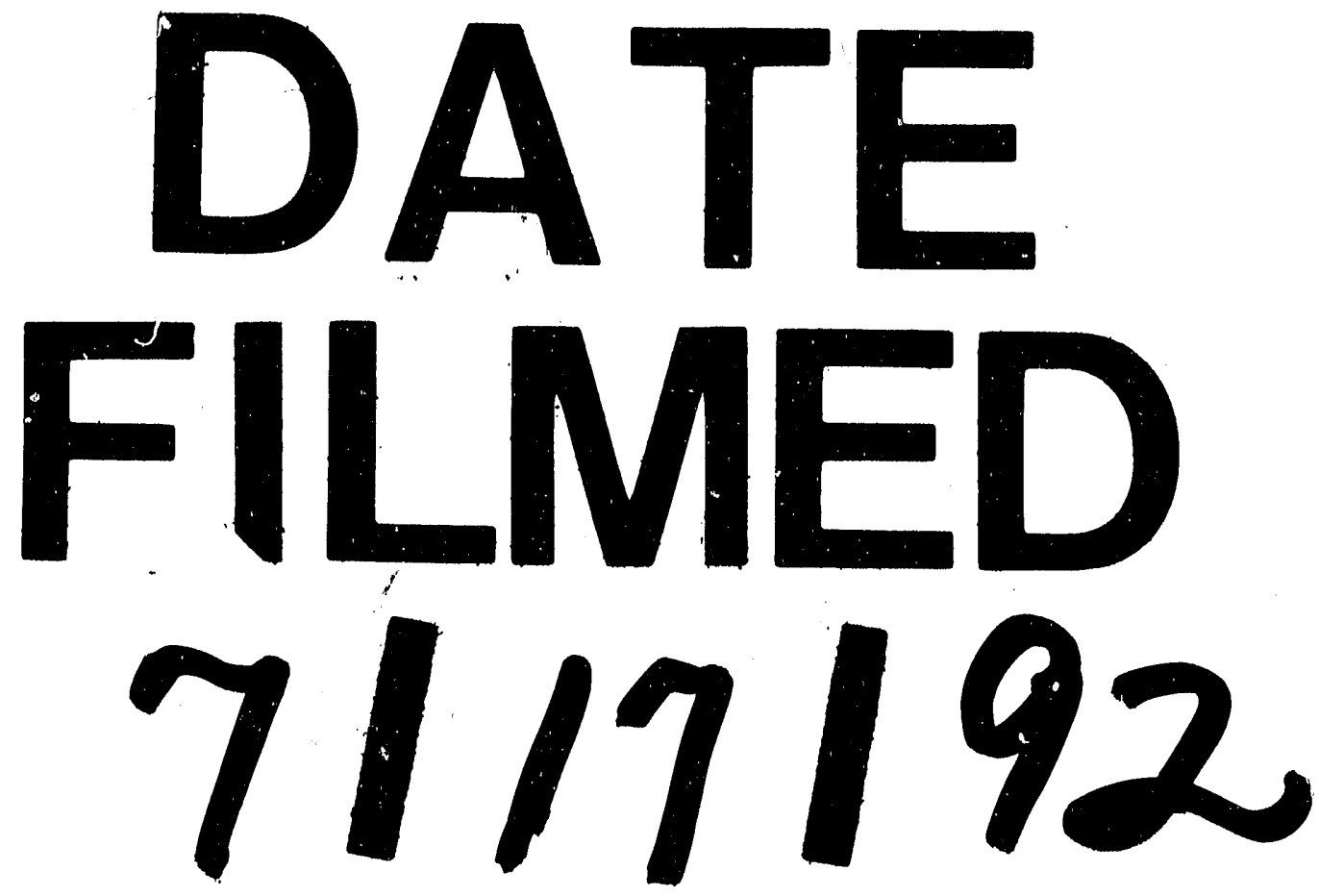
